\title{
因HAD
}

ISSN-L: 2530-5115

DOI: http://doi.org/10.22585/hospdomic.v3i2.57

\section{Comunicar la ciencia}

\section{Communicate science}

\author{
María Sanz-Lorente ${ }^{1,2}$, Rocio Guardiola-Wanden-Berghe ${ }^{3,4}$ \\ 1. Centro de Salud Pública de la Consellería de Sanidad Universal y Salud Pública. Manises, España. \\ 2. Universidad Miguel Hernández, Departamento de Salud Pública e Historia de la Ciencia, Sant Joan \\ d'Alacant, España. \\ 3. Consorci Parc de Salut Mar, Instituto de Neuropsiquiatría y Adicciones (INAD), Barcelona, España. \\ 4. Centro de Investigación Biomédica. Barcelona, España.
}

\section{Correspondencia/Correspondence}

María Sanz-Lorente

Centro de Salud Pública de la Consellería de

Sanidad Universal y Salud Pública. Manises,

España.

msanzlor@gmail.com

Recibido/Received

19.10.2018

Aceptado/Accepted

18.02.2019
Conflicto de Intereses/Competing interest

Las autoras declaran la inexistencia de conflicto de interés.

CÓMO CITAR ESTE TRABAJO | HOW TO CITE THIS PAPER

Sanz-Lorente M, Guardiola-Wanden-Berghe R. Comunicar la ciencia. Hosp Domic. 2019;3(2): 173-83. 


\section{RESUMEN}

La comunicación es esencial a la naturaleza y práctica de la ciencia. Los científicos no sólo comunican los resultados a sus colegas, sino que también se apoyan en el conocimiento de trabajos anteriores para formular propuestas y metodologías de investigación.

Esta comunicación puede definirse como el proceso de presentación, almacenamiento, distribución y recepción de la información científica en la sociedad. Es decir, es el conjunto de actividades que interpretan y hacen accesible el conocimiento científico al público general. Por tanto, todas aquellas labores que llevan el saber científico a las personas interesadas en entender o informarse de ese tipo de conocimiento.

Se puede deducir claramente que Internet, y sobre todo la World Wide Web (www), es una potente herramienta para la divulgación de la ciencia, a pesar de ello sigue siendo ineludible la evaluación de la calidad de la información que se trasmite.

Palabras clave: Comunicación; Comunicación en Salud; Acceso a la Información; Tecnología de la Información; Almacenamiento y Recuperación de la Información; Gestión del Conocimiento para la Investigación en Salud.

\section{ABSTRACT}

Communication is essential to the nature and practice of science. The scientists not only to communicate the results to their colleagues, they also rely on the knowledge of previous works to formulate proposals and research methodologies.

This communication can be defined as the process of presentation, storage, distribution and receipt of scientific information in society. I.e., is the set of activities that interpret and make accessible scientific knowledge to the general public. Therefore, all those tasks that carry the scientific knowledge to people interested in understanding or finding out about that kind of knowledge.

We can clearly deduce that Internet, and especially the World Wide Web (www), is a powerful tool for the divulgation of science, nevertheless the evaluation of the quality of the information transmitted remains unavoidable.

Keywords: Communication; Health Communication; Access to Information; Information Technology; Information Storage and Retrieval; Knowledge Management for Health Research. 


\section{INTRODUCCIÓN}

La ciencia se basa en la aportación de los hallazgos y descubrimientos precedentes y en su integración en un conjunto de conocimientos compartidos por los científicos y que constituye su paradigma. Puede dividirse estructuralmente en tres esferas: producción, comunicación y utilización de los conocimientos científicos. No se puede concebir la ciencia sin que haya comunicación y transmisión de conocimientos.

La comunicación es esencial a la naturaleza y práctica de la ciencia. Los científicos no sólo comunican los resultados a sus colegas, sino que también se apoyan en el conocimiento de trabajos anteriores para formular propuestas y metodologías de investigación. El intercambio de opiniones y datos con los pares es parte esencial de la fase experimental. Por lo tanto, la comunicación está presente en todas las etapas de la investigación.

Ahora bien, una de las características propias del conocimiento científico es que debe ser público y consensuado. Es decir, la ciencia debe ser comunicable; si un cuerpo de conocimiento no lo es, entonces por definición no es científico. La comunicabilidad es a su vez una condición necesaria para la verificación de los datos empíricos y de las hipótesis científicas $(1,2)$.

La comunicación de la ciencia puede definirse como el proceso de presentación de la información científica en la sociedad. Podría también utilizarse la definición de comunicación académica (3): «El estudio de cómo los académicos en cualquier campo, utilizan y difunden la información a través de los canales formales e informales».

Los canales formales corresponden a la información publicada (es decir, hecha pública), como la que contienen los libros o los artículos de revista, y que suele estar disponible durante largos períodos para un amplio público. Los canales informales son más efímeros y están limitados a ciertos destinatarios. Ejemplos notables de éstos son la comunicación oral, la correspondencia personal, las actas de reuniones científicas, las conferencias y, actualmente, los documentos prepints (versiones de documentos no evaluados o antes de serlo que generalmente se presentan en formato digital).

Los medios informales se distinguen de los canales formales en que permiten una interacción más inmediata entre el emisor de la información y el receptor (4). Este tipo de comunicación tiene importancia en todas las áreas de la actividad científica y en todos los estratos de la comunidad científica. Sin embargo, su peso y significado no son homogéneos y tiene unas características que le son propias (5); ver figura1. 
Figura 1. Esquema de la comunicación de la ciencia

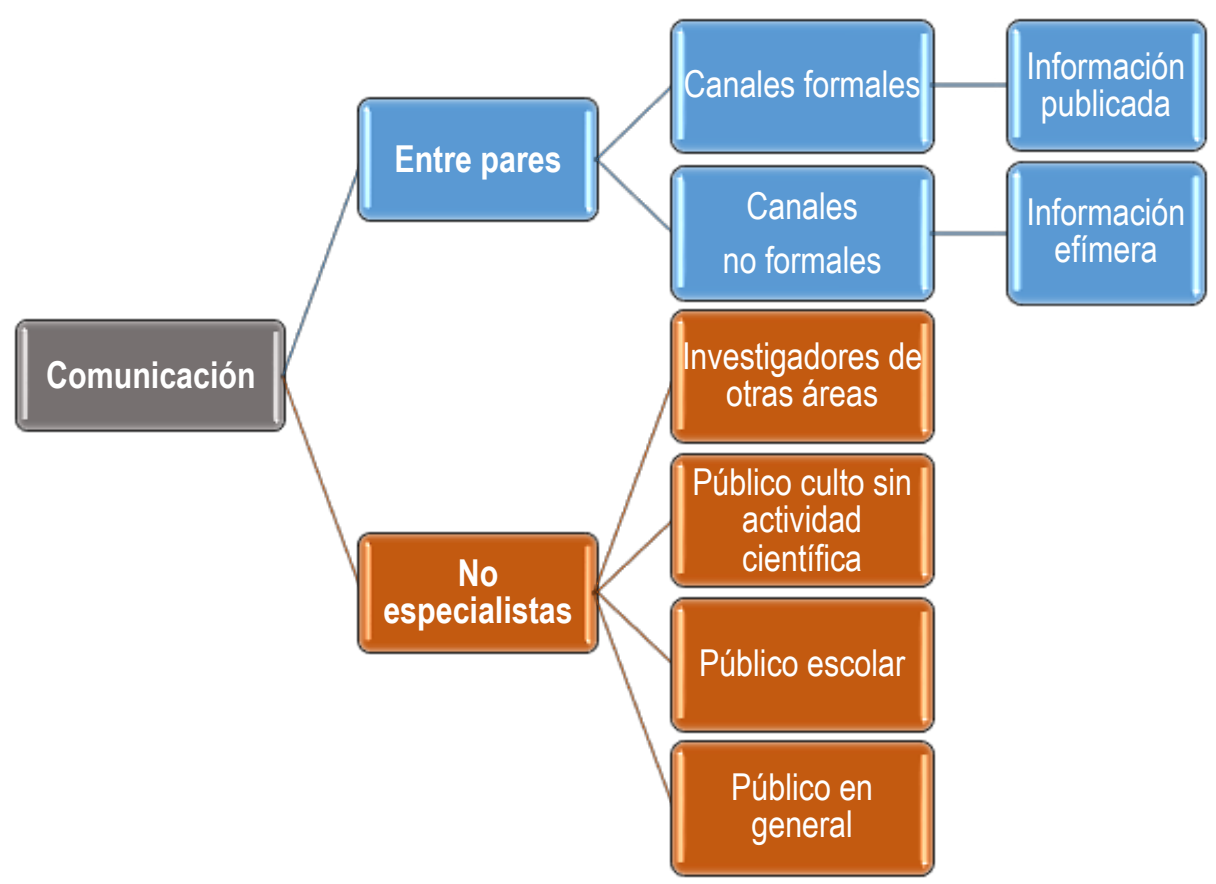

Ahora, al presente, las comunicaciones formales e informales están experimentando alteraciones radicales, de tal modo que la distinción entre ambas se vuelve cada vez más borrosa. Este desdibujarse de las divisiones establecidas es un elemento clave en el cambio de los medios impresos a los electrónicos. Los papeles tradicionales asignados al productor, al procesador (o editor) y al usuario están experimentando grandes transformaciones (4). Por ejemplo, la autopublicación de un informe de investigación en la Red por parte de un científico, o de su institución, no puede ser definida con claridad en términos de la tradicional división de comunicación formal/informal, puesto que están implicados ambos tipos de acciones. Informal, porque la comunicación no es parte de una publicación 'clásica', y formal, por cuanto la comunicación no está limitada a un grupo definido de receptores, sino disponible para cualquiera que desee tener acceso a ella.

La comunicación informal, que quizá representa los procesos menos estructurados de comunicación entre científicos, conoce actualmente un auge debido a las facilidades de los medios electrónicos. La comunicación, como discurso informal, mediatizada por el correo electrónico, los grupos de discusión y las redes sociales, abarca un público más amplio y permite reunir al mundo científico al menos en el ciberespacio.

Las tecnologías de la comunicación y la información (TIC) están influyendo en la estructura de la comunicación científica. Por esto, los modelos tradicionales de edición y comunicación académica deben estar sujetos permanentemente a nuevos análisis en el contexto del panorama actual.

De igual forma, como comunicación de la ciencia se puede distinguir, según la línea de transmisión (emisor - mensaje - receptor), entre divulgación, difusión y diseminación; ver figura 2. 


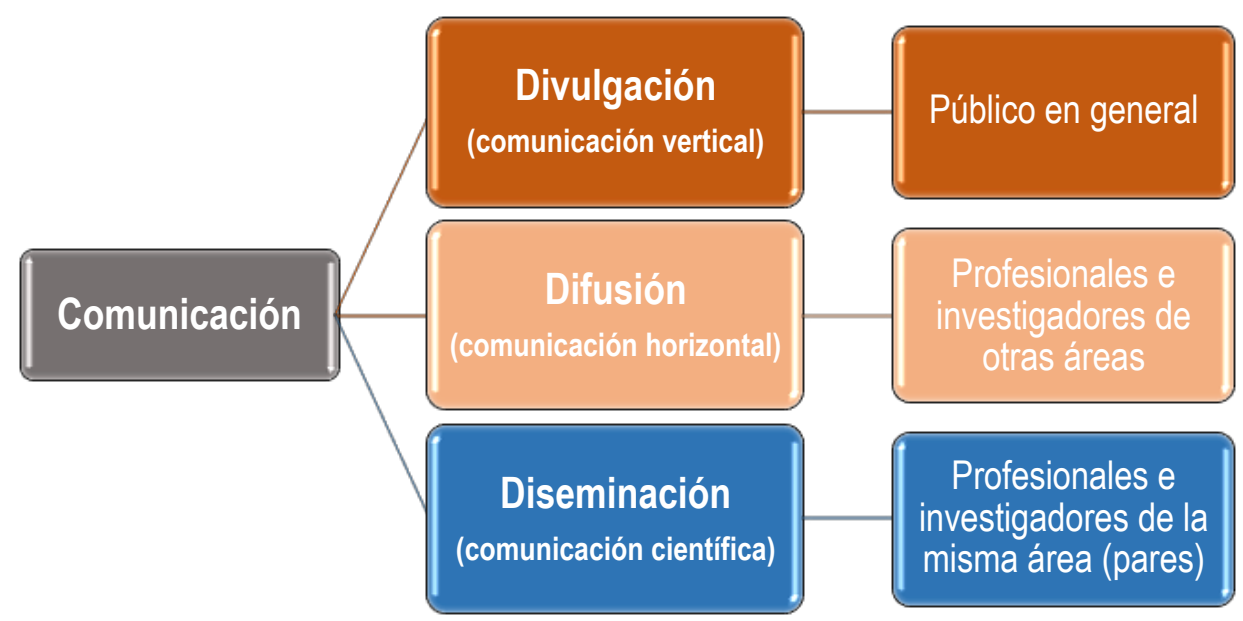

Divulgación sería el envío de mensajes elaborados mediante trans-codificación de lenguajes especializados a lenguajes omnicomprensibles.

Se parte, en general, de que el mensaje se dirige a un público formado por personas de muy distinta preparación. El uso más frecuente se refiere a la comunicación del científico con el público en general, por lo que esta modalidad puede llamarse "comunicación vertical». La actual especialización de la ciencia y de la tecnología hace que la comunicación entre científicos de distintas disciplinas se considere también una labor de divulgación.

Difusión es la misión del investigador de trasmitir al público los conocimientos sobre su disciplina. El público incluye a los profesionales de otras áreas. La difusión se confunde con la acción de informar, no existiendo una decodificación del lenguaje críptico. Es frecuente emplear la palabra difusión cuando se hace referencia a la comunicación entre personas agrupadas por motivos profesionales o por intereses específicos. Por ello, se ha calificado como "comunicación horizontal».

Diseminación es el envío de mensajes elaborados, en lenguajes especializados, a perceptores selectivos y restringidos. La diseminación científica, referida generalmente como "comunicación científica» o "comunicación entre pares», es la trasmisión de datos e informaciones para especialistas del mismo sector de la ciencia, en lenguaje específico.

La clasificación de la comunicación de la ciencia en las tres categorías enunciadas dependerá, tomando como base la tradicional concepción de la comunicación (emisor - mensaje - receptor), del emisor y del receptor, pero también de la forma y estructura del mensaje (6).

Desde estas premisas, queda claro que las TIC pueden ser una excelente herramienta para favorecer la propagación de la información y la democratización del conocimiento. Pero, también es cierto que favorecen, más si cabe, la confluencia entre ellas. Estas tecnologías permiten la expansión y la permeabilidad del saber a un bajísimo coste, impulsarlas es pues esencial. Se trata de una precondición ineludible si de verdad se apuesta por la plena incorporación en la sociedad de la información, donde las TIC pueden ayudar técnicamente a la mejora de los canales de información, comunicación, deliberación y participación de los ciudadanos en la toma de decisiones (7).

Los conceptos de información y de comunicación se han trasmutado al haberse modificado las dimensiones del tiempo y del espacio sobre los que se han sostenido. Si algo define este nuevo escenario es la velocidad con la que la información se genera, se transmite y se procesa (8). 


\section{DIVULGACIÓN CIENTÍFICA}

Es el conjunto de actividades que interpretan y hacen accesible el conocimiento científico al público general, es decir, todas aquellas labores que llevan el saber científico a las personas interesadas en entender o informarse de ese tipo de conocimiento. La divulgación pone su interés no sólo en los descubrimientos científicos del momento, sino también en teorías más o menos bien establecidas o aceptadas socialmente (por ejemplo, la teoría de la evolución) o incluso en campos enteros del conocimiento científico.

Pero, hay que dejar patente que comunicar es mucho más que informar; se puede informar (dar noticia de algo) sin comunicar. Para comunicar es necesario ser capaces de trasmitir adecuadamente el mensaje y compartir los conocimientos con el público no especializado, poniéndolo a un nivel de asimilación adecuado. Sólo la divulgación permite extender los conocimientos a un público no especialista.

Etimológicamente, comunicación procede del latín communicare, es decir, compartir o poner en común. Por eso, la comunicación no debería ser diseñada como un suministro de información unidireccional, sino como una oportunidad de poner ideas en común.

En definitiva, podemos superar los equívocos si por información entendemos el contenido de la comunicación y si concluimos que, dada la profunda grieta cultural que existe entre los científicos y tecnólogos y el público general, para comunicar informaciones de carácter científico es preciso que la comunicación se lleve a cabo divulgando sus contenidos.

De todas formas, existen otras opiniones según las cuales la palabra información sería más adecuada para expresar la comunicación de las novedades científicas mientras que la divulgación se correspondería mejor con la comunicación de la ciencia que ya existe (9). Tener en cuenta que el concepto "divulgación científica» está recibiendo otras denominaciones, como: comunicación científica pública, popularización de la ciencia o comunicación social de la ciencia.

Por otra parte, no se debe olvidar que el conocimiento científico además de ser "cierto" y «probado" y de "aportar nuevas ideas", tiene que ser provechoso para el progreso de la sociedad. La sociedad es el fin último del trabajo investigador y es a la sociedad a la que de forma directa o indirecta va dirigido (10).

Una vez admitido que la comunicación entre la persona investigadora y la sociedad, e incluso entre personas investigadoras de diferentes disciplinas científicas es esencial, se trata de explicar «de forma clara y concisa» (mediante un proceso de simplificación de las ideas, uso de palabras comunes, utilización de imágenes y audiovisuales que capten la atención, etc.) a una audiencia lo más amplia posible, los temas relevantes de un trabajo para aumentar la valoración social del mismo. Hay que tener presente que no se está ante un público único.

La UNESCO reconoció, en la Declaración de la Ciencia y el Uso del Conocimiento Científico, adoptada en la Conferencia Mundial de la Ciencia de 1999 (11), la importancia de que los científicos se involucren activamente en la comunicación con el público. En concreto, la declaración afirma:

- El conocimiento científico debe ser compartido.

- Se necesita que haya una auténtica cooperación entre gobiernos, sociedad civil, sector empresarial y científicos.

- Los científicos deben regirse por los estándares éticos correspondientes.

Contrariamente a estas recomendaciones, muchos científicos nunca han hecho el esfuerzo de compartir sus conocimientos fuera de su entorno académico, pues no creen que deban ser ellos quienes lo hagan. Algunos han intentado en ocasiones dirigirse al público directamente o a través de los medios de comunicación y no han tenido éxito (puede ser que no se prepararan adecuadamente para esta empresa). Finalmente, hay un tercer grupo de científicos que han hecho de la 
comunicación social una parte más de su actividad profesional, resultándoles esta tarea altamente satisfactoria (12).

\section{DIVULGACIÓN CIENTÍFICA EN INTERNET}

La necesidad de hacer público el conocimiento científico se formaliza con la Revolución Científica en el siglo XVII y se mantiene hasta el momento actual, debido, entre otros factores, a la progresiva institucionalización y profesionalización de la ciencia, especialmente desde mediados del siglo XIX (13).

No obstante, para facilitar la divulgación de la ciencia, en este momento, las potencialidades de la Web 2.0 son el mayor medio de comunicación y difusión que existe.

El desarrollo de la sociedad de la información es innegable. Se puede decir que nos estamos refiriendo al resultado de una serie de procesos de cambios tecnológicos, organizativos, económicos, sociales e institucionales que están alterando las relaciones de producción y consumo, los hábitos de trabajo, los estilos y la calidad de vida y las relaciones entre los diferentes actores públicos y privados de nuestra sociedad (14). Estos pasos han desembocado en el desarrollo de la potencialidad de Internet y en la 'supuesta' disponibilidad universal de los conocimientos (15). Aunque, persiste un problema, el desconocimiento de las herramientas de difusión a través de Internet es algo bastante habitual entre la población e incluso en las personas investigadoras, que no suelen percibir la importancia y necesidad de las mismas. Ahora bien, la información que se difunde en la Red debe estar preparada para que se encuentre, y especialmente para que la encuentren los buscadores más utilizados. El uso de Google, por ejemplo, como herramienta de búsqueda y de acceso a la información de todo tipo (incluida la que no se busca) ha dado un vuelco a los hábitos informativos de las personas, tanto para obtener información como para difundirla. En consecuencia, alfabetizar científicamente a la sociedad es poner las bases para provocar un mayor interés del público por la investigación y sus logros; y así, de paso, reducir las reacciones negativas y poco justificadas que a veces provocan estos avances. Significa también capacitar a los ciudadanos para que puedan opinar, con conocimiento de causa, si ello fuese necesario, en decisiones de política científica que a todos nos afectan. Esta alfabetización, lejos de ser un lujo, es una urgente necesidad (14).

En esta etapa de transición hacia el paradigma informacional, Internet ha llegado para quedarse y ha revolucionado la forma en que la población recibe la información. En esta tarea informativa, pero también con un fuerte componente educativo, las herramientas 2.0 (blogs, wikis y redes sociales) se han convertido en un nuevo medio que permite a más personas llegar a una buena información generada por un sistema cooperativo. Habilita a los usuarios para ser participantes activos coproduciendo contenidos, aumentando la conectividad y la colaboración en la producción de nuevo conocimiento (16).

Probablemente, la Red ha puesto en cuestión una de las funciones básicas de los medios tradicionales y de sus profesiones asociadas: la mediación profesional de los comunicadores en los procesos de acceso del público a las fuentes del saber. Este nuevo paradigma multiplica el número de voces, pero a la vez diluye su autoridad al haber fracturado el sistema de control erudito previo a la difusión pública de información. Con todo, conecta a la población a un espacio comunicativo universal.

Afortunadamente, Internet es una plataforma esencial para la divulgación científica. A día de hoy, a través de Internet puedes llegar a mucha gente, incluso a aquella que no está interesada directamente en la ciencia o que no sabe que la ciencia es interesante. Para ello, la Web 2.0 es una 
herramienta que puede ser muy útil para que los investigadores puedan dar a conocer sus resultados a la población y puedan interaccionar directamente con las personas interesadas.

Un artículo publicado en la revista Science (17) probaba que la divulgación científica no está logrando despertar el interés de la población general.

Seguramente, la gran cantidad de información disponible en Internet hace que sea difícil llegar a una audiencia no especializada y sean los resultados de la búsqueda en Google, y otros motores de búsqueda, lo que determine en gran parte lo que en última instancia encuentra el internauta cuando busca información en la Red. De este modo, es complicado llegar a públicos que no consumen habitualmente Webs específicas de ciencia.

Internet ha provocado un efecto paradójico en la comunicación del conocimiento, que por una parte se ha desprofesionalizado (publica cada vez más gente sin una sólida formación) y por otra parte se ha súper especializado fragmentando el conocimiento en infinidad de parcelas. La contradicción es que Internet es la gran oportunidad pero no se está sabiendo aprovechar, la forma de consumir la información por los internautas choca con la rigidez del mensaje académico. Salvo excepciones de usuarios con suficiente criterio previo, la mayoría utilizan un motor de búsqueda para encontrar información sin fijarse en cuál es la fuente que hay detrás y su credibilidad científica. Los algoritmos de posicionamiento utilizados por Google y otros motores de búsqueda, están determinando en gran parte la información ofertada en la Red. Sin embargo, como ya se ha mencionado, no existen aún suficientes científicos que se impliquen en la divulgación de la ciencia a través de Internet y en su estudio, aunque es esperable que su número vaya aumentando poco a poco.

La "e-Comunicación», como nuevo escenario de la comunicación pública en la era de Internet, ha de interpretarse no de un modo apocalíptico, sino como la ocasión para redefinir el perfil y las exigencias de los investigadores, así como los contenidos y los procedimientos de su formación académica.

Quizá, conocer los motores de búsqueda y que hoy por hoy son el punto de partida más común para buscar información, ofreciendo entre sus resultados mejor posicionados los artículos de la Wikipedia $(18,19)$, podría ser el primer paso para entender que esta wiki se presenta como una valiosa herramienta para la divulgación de la información científica (20). Su elevado número de consultas, junto con la facilidad y rapidez de la actualización de los contenidos la dotan de un enorme potencial como herramientas para la trasmisión del conocimiento, permitiendo que cualquier persona investigadora difunda sus propios trabajos sin prácticamente ningún intermediario.

La participación de los expertos en la actualización y difusión del conocimiento en beneficio de la sociedad, que ya se aprecia en algunas áreas, debería generalizarse, aunque sería deseable que contara con el adecuado reconocimiento académico-profesional.

Es básico que el mundo científico asuma la comunicación pública en la Red como una estrategia de su labor, e incluso que utilice las herramientas que le brinda la Web 2.0 para informar directamente al público. Hoy hay que ir mucho más allá de las formas tradicionales de compartir y socializar el conocimiento; por ello comunicar en red se ha convertido en una estrategia esencial, complementaria e indisociable, del cogito ergo sum que ha sido la generación del conocimiento científico $(21,22)$.

\section{CALIDAD DE LA INFORMACIÓN EN LA WEB}

Internet se ha convertido en una tecnología con enorme crecimiento y penetración con más de 4.156 usuarios, a 31 de diciembre de 2017, según datos de «Internet World Stats»; http://www. internetworldstats.com/). 
Estos datos junto a la enorme facilidad, técnica y económica, para la creación y almacenamiento de documentos en la Red supone una importante ventaja que no está exenta de riesgos e inconvenientes. Este problema puede verse muy agravado por la heterogeneidad de la calidad de dicha información, ya que en demasiadas ocasiones desconocemos el origen y su veracidad. Por tanto, es necesaria la evaluación rigurosa y estricta de la información recabada.

La evaluación de la calidad es una etapa muy importante en el proceso de búsqueda de información, pero a menudo no se le presta la suficiente atención. Algunas premisas que pueden tenerse en cuenta en este proceso de evaluación, serían:

- Examinar los datos para determinar por qué han sido recuperados los registros (analizar los posibles problemas de cada etapa del proceso de búsqueda).

- Calcular el porcentaje de registros relevantes y/o pertinentes.

- Reflexionar críticamente sobre el contenido de la información obtenida.

- Considerar la idoneidad de las fuentes de donde se obtuvo la información.

Dentro de este contexto, surge la necesidad de realizar mediciones relacionadas con la calidad de una sede Web. El peritaje de la calidad en la Web (páginas, blogs, etc.) no es una tarea sencilla, no existiendo una definición clara del concepto de calidad de información en la Web y se carece de un marco teórico bien fundamentado sobre la evaluación de esta calidad (23). Se podría decir, que la calidad de un sitio Web es todavía un constructo sin definir, y existen numerosas interpretaciones relacionadas con dicho concepto.

La mayoría de los sistemas existentes de evaluación se basan en el establecimiento de una serie de criterios (indicadores). Si bien, uno de los aspectos que deberían considerar estas herramientas de evaluación es permitir a los usuarios poder obtener sus propias conclusiones.

Con el fin de facilitar la tarea de comprobación de la calidad Web, existen estudios que han intentado simplificar y unificar las variables a testar. Por ejemplo, Lopes (24) concentró en 7 categorías los indicadores de calidad con el fin de determinar la credibilidad de la información sobre salud en la Web. Posteriormente, esta misma autora propuso que para observar patrones de calidad sería necesario utilizar criterios de credibilidad del sitio Web y también los de contenido (25).

A partir de estas propuestas, Guardiola-Wanden-Berghe et al (26), refundieron en 22 ítems las variables sobre calidad Web aportadas por las principales instituciones, demostrando a su vez una correlación positiva entre el cumplimiento de las variables de calidad y el Indicador de Credibilidad (8 ítems). Esta correspondencia daba al usuario general la posibilidad de valorar la calidad de una determinada Web utilizando tan solo 8 variables de fácil comprensión (27).

Partiendo de que la experiencia del usuario resume todo el conjunto de percepciones que se acumulan durante la visita a un sitio Web, convendría desarrollar sistemas que analicen la calidad de información percibida por este. Sin embargo, la evaluación de la calidad en los sitios Web basada en la opinión que aportan los usuarios es una tarea de gran complejidad que no ha sido lo suficientemente contrastada (28). Es evidente que disponer de indicadores para aplicar en el proceso de evaluación es, sin lugar a duda, necesario. Pero, muchas veces los mismos son de difícil comprensión para aquellas personas no expertas en la materia y que, al fin y al cabo, son los usuarios finales de toda esta ingente información disponible en la Red.

Por lo expuesto en este apartado, se puede deducir claramente que Internet, y sobre todo la World Wide Web (www), es una potente herramienta para la divulgación de la ciencia, a pesar de ello sigue siendo ineludible la evaluación de la calidad de la información que se trasmite. 


\section{BIBLIOGRAFÍA}

1. Ziman J. El conocimiento público: un ensayo sobre la dimensión social de la ciencia. México DF, México: Fondo de Cultura Económica; 1972.

2. Bunge M. La ciencia: su método y su filosofía. Buenos Aires, Argentina: Siglo Veinte; 1966.

3. Meadows A. Communicating Research, Library and Information Science. San Diego, USA: Academic Press; 1998.

4. Russell JM. Scientific communication at the beginning of the 21st century. Int Soc Sci J. 2001;53(168):271-82. DOI: 10.1111/1468-2451.00314

5. López Piñero J, Terrada M. Los indicadores bibliométricos y la evaluación de la actividad médico-científica (II): La comunicación científica en las distintas áreas de las ciencias médicas. Med Clín (Barc). 1992;98(3):101-6.

6. Bucchi M. Communicating science. In: Bucchi M, editor. Science in society. New York, USA: Routledge; 2004. p. 107-23.

7. Colombo C. Innovación democrática y TIC, ¿hacia una democracia participativa? Rev Internet Derecho Política. 2006;(3):28-40.

8. Sanz-Valero J, Castiel L. Las aventuras de Alicia en el maravilloso mundo del conocimiento: el camino hacia la actual alfabetización. Hist Cienc Saude Manguinhos. 2010;17(1):153-64. DOI: 10.1590/S0104-59702010000100010; PMID: 21461500

9. Fundación Cotec para la Innovación Tecnológica. Comunicar la ciencia. Madrid, España: Fundación Cotec para la Innovación Tecnológica; 2006.

10. García Álvarez de Toledo J, Fernández Sánchez R. Difusión y divulgación científica en Internet. Oviedo, España: Gobierno del Principado de Asturias; 2011.

11. Organización de las Naciones Unidas para la Educación, la Ciencia y la Cultura (UNESCO). Declaración de la Ciencia y el Uso del Conocimiento Científico, adoptada en la Conferencia Mundial de la Ciencia de 1999 [monografía en Internet]. Budapest, Hungría: UNESCO; 1999 [citado 8 de julio de 2018]. Disponible en: http://www.unesco.org/science/wcs/esp/declaracion_s.htm

12. De Semir V, Revuelta G. La importancia de la comunicación en el entorno científico. En: Serés E, Rosich L, Bosch F, editores. Cuadernos de la Fundación Dr Esteve No 20: Presentaciones orales en biomedicina. Barcelona, España: Fundación Dr. Antonio Esteve; 2010. p. 1-7.

13. Martín-Rodero H, Sanz-Valero J, Galindo-Villardón P. The methodological quality of systematic reviews indexed in the MEDLINE database: A multivariate approach. The Electronic Library. 2018;36(1):146-58. DOI: 10.1108/EL-01-2017-0002

14. Lozano L. Monografía sobre las regiones y el desarrollo de la sociedad de la información. En: Alaban A, editor. La Unión Europea frente al desarrollo de la Sociedad de Información en las Regiones. La Coruña, España: Diputación provincial; 2000. p. 33-92.

15. Castiel L, Sanz-Valero J. Política científica: manejar la precariedad de los excesos y desnaturalizar la ideología "publicacionista" todopoderosa. Salud Colectiva. 2009;5(1):5-11.

16. De Semir V, Revuelta G. Información médica y educación sanitaria en la revolución de las TIC. En: De Semir V, Revuelta G. Cuadernos de la Fundación Dr. Antonio Esteve no 25: El periodismo biomédico en la era 2.0. Barcelona, España: Fundación Dr. Antonio Esteve; 2012. p. 1-8.

17. Brossard D, Scheufele A. Science, new media, and the public. Science. 2013;339(6115):40-1. DOI: 10.1126/science.1232329 
18. Laurent M, Vickers T. Seeking health information online: does Wikipedia matter? J Am Med Inform Assoc. 2009;16(4):471-9. DOI: 10.1197/jamia.M3059; PMID: 19390105

19. Sanz-Valero J, Wanden-Berghe C, Culebras-Fernández JM, Gil A, Ruiz MD, Luengo LM, et al.; CDC-Nut SENPE. Wikipedia and wikinutrition: key tools for the global promotion of nutrition. Nutr Hosp. 2012;27(5):1375-9. DOI: 10.3305/nh.2012.27.5.5984; PMID: 23478681

20. Heilman JM, Kemmann E, Bonert M, Chatterjee A, Ragar B, Beards GM, et al. Wikipedia: a key tool for global public health promotion. J Med Internet Res. 2011;13(1):e14. DOI: 10.2196/ jmir.1589; PMID: 21282098

21. Editorial. Geting the world out. Nat Neurosci. 2009;12(3):235. DOI: 10.1038/nn0309-235; PMID: 19238179

22. Brumfiel G. Science journalism: Supplanting the old media? Nature. 2009;458(7236):274-7. DOI: 10.1038/458274; ; PMID: 19295582

23. Gertz M, Ozsu M, Saake G, Sattler K. Report on the Dagstuhl Seminar "Data quality on the Web". SIGMOD Record. 2004;33(1):127-32. DOI: 10.1145/974121.974144

24. Lopes IL. Novos paradigmas para avaliação da qualidade da informação em saúde recuperada na Web. Ciênc Informação. 2004;33(1):81-90. DOI: 10.1590/S0100-19652004000100010

25. Lopes IL. Criterios de qualidade para avaliaçao da informaçao na World Wide Web. Brasilia, Brasil: Editora do Departamento de Ciencia da Informaçao da Universidade de Brasilia; 2007.

26. Guardiola-Wanden-Berghe R, Gil-Pérez JD, Sanz-Valero J, Wanden-Berghe C. Evaluating the quality of websites relating to diet and eating disorders. Health Inf Libr J. 2011;28(4):294-301. DOI: 10.1111/j.1471-1842.2011.00961.x; PMID: 22051128

27. Sanz-Lorente M, Guardiola-Wanden-Berghe R. Evaluación de la calidad de las páginas Web sobre el Hospital a Domicilio: el Indicador de Credibilidad como factor pronóstico. Hosp Domic. 2017; 1(2):73-82. DOI: 10.22585/hospdomic.v1i2.11

28. Eysenbach G, Powell J, Kuss O, Sa ER. Empirical studies assessing the quality of health information for consumers on the World Wide Web: a systematic review. JAMA. 2002;287(20):2691- 700. DOI: 10.1001/jama.287.20.2691; PMID: 25996397 